
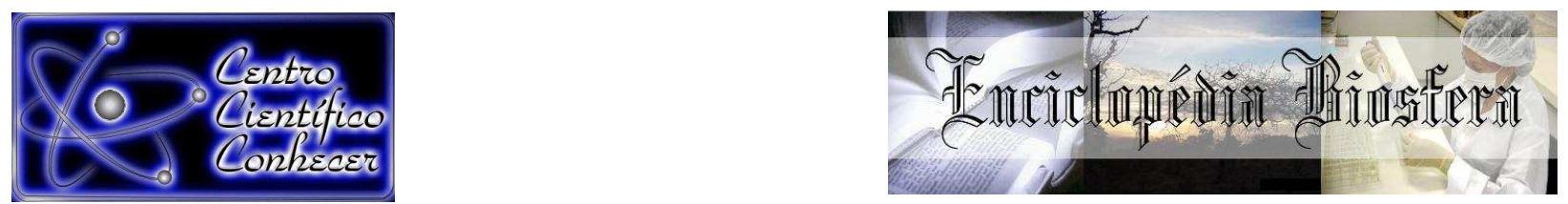

\title{
APLICAÇÃO DO MÉTODO PBC PARA AVALIAÇÃO DA COMPLEXIDADE DAS DISTRIBUIÇÕES GAMA E NAKAGAMI NA ANÁLISE DE DADOS DE PRECIPITAÇÃO
}

\author{
Larissa Bueno Fernandes ${ }^{\prime}$, Josmar Mazucheli ${ }^{1}$, Isabele Picada Emanuelli, ${ }^{2,3}$ \\ ${ }^{1}$ Universidade Estadual de Maringá/UEM, Maringá, Brasil \\ (jmazucheli@gmail.com) \\ ${ }^{2}$ Centro Universitário de Maringá/UniCesumar, Maringá, Brasil. \\ ${ }^{3}$ Instituto Cesumar de Ciência, Tecnologia e Inovação/ICETI Maringá, Brasil. \\ Recebido em: 02/10/2017 - Aprovado em: 21/11/2017 - Publicado em: 05/12/2017 \\ DOI: 10.18677/EnciBio_2017B102
}

\begin{abstract}
RESUMO
Em geral, a seleção da distribuição de probabilidade com o melhor ajuste não considera a complexidade das distribuições rivais. O método PBC (Parametric Bootstrap Cross-Fitting) mede o viés causado pelo mimetismo das distribuições candidatas, levando em consideração a avaliação da complexidade das mesmas. Considerando a importância de explicar o comportamento dos volumes de precipitações mensais e da possibilidade do uso do método PBC para comparação do ajuste de duas distribuições concorrentes, objetivou-se neste trabalho realizar a discriminação e a comparação do mimetismo das distribuições Gama e Nakagami, aplicando o método PBC aos dados de precipitações mensais da estação meteorológica convencional de Maringá - PR, observados entre 1964 e 2016. Embora Nakagami não seja uma distribuição muito utilizada em estudos envolvendo variáveis de natureza climatológica, os resultados referentes a aplicação do método PBC indicam que para a maior parte das séries históricas mensais, a distribuição mais apropriada para a descrição do comportamento da precipitação mensal é a Nakagami quando comparada a Gama. Ainda, foi verificado que a Gama é funcionalmente mais complexa em relação a Nakagami, apresentando maior viés causado pelo mimetismo em todas as séries de precipitação mensal total.
\end{abstract}

PALAVRAS-CHAVE: Climatologia; Distribuição de Probabilidade; Método CrossFitting;

\section{APPLICATION OF THE PBC METHOD FOR THE EVALUATION OF COMPLEXITY OF THE GAMA AND NAKAGAMI DISTRIBUTIONS IN THE ANALYSIS OF PRECIPITATION DATA}

\begin{abstract}
In general, the selection of the probability distribution with the best fit does not take into account the distributions' complexity. The PBC (Parametric Bootstrap CrossFitting) method quantifies the bias due to the mimicry of the candidate distributions, taking into account the evaluation of the models' complexity. Considering the importance of explaining the behavior of the monthly rainfall volumes and the possibility of using the PBC method to compare the fit of two competing distributions, the objective of this work is to discriminate and compare the mimicry of the Gamma and Nakagami distributions, by applying the PBC method to the monthly precipitation
\end{abstract}


data of the Maringá - PR meteorological station, observed between 1964 and 2016. Although Nakagami is not a very usual distribution in studies involving climatological variables, results referring to the PBC method indicated that for most of the monthly historical series, Nakagami is the most appropriate distribution for the description of monthly precipitation behavior when compared to Gamma. In addition, it was observed that the Gamma distribution was shown to be functionally more complex than the Nakagami, presenting greater measures of bias due to mimicry for all datasets.

KEYWORDS: Climatology; Cross-Fitting Method; Probability Distribution.

\section{INTRODUÇÃO}

A caracterização da distribuição da precipitação constitui um importante instrumento de apoio para estudos e desenvolvimento de atividades econômicas relacionadas. $O$ estudo da distribuição de variáveis climáticas determina padrões de ocorrência permitindo uma previsão razoável do comportamento climático pluvial de uma região (DOURADO NETO et al., 2005). Tanto a intensidade quanto a frequência com que os fenômenos climáticos ocorrem influenciam diretamente na sustentabilidade ambiental e econômica dos setores do agronegócio (BEYRUTH, 2008).

No estudo de variáveis climatológicos (volume de precipitação, velocidade do vento, temperatura, entre outras), em geral, existe o interesse na estimação da probabilidade de uma variável aleatória $X$ exceder um valor $x_{T}$ qualquer, ou seja $P\left(X \geq x_{T}\right)$. Em climatologia, a estimação desta quantidade é o foco principal do que é conhecido como análise de frequências. O objetivo da análise de frequências é relacionar a magnitude de eventos extremos com a frequência de ocorrência por intermédio de uma distribuição de probabilidade apropriada (CHOW et al., 2013).

$\mathrm{Na}$ literatura, várias distribuições de probabilidade são usadas no estudo de variáveis climatológicas. Dentre estas citam-se: Gama, Weibull, Log-Normal, LogLogística, Inversa-Gaussiana e Valor-Extremo (COE; STERN, 1982). Dado um conjunto de observações, a seleção de uma distribuição é, sem dúvida, o passo mais crítico, e aquele que apresenta as maiores incertezas (MEYLAN et al., 2012).

Outra distribuição que pode ser aplicada para dados climatológicos é a Nakagami cujas aplicações concentram-se, principalmente, na área de engenharia de comunicações (NAKAGAMI, 1960). Se comparada com outras distribuições, a Nakagami é considerada genérica, de grande flexibilidade e simplicidade matemática. O número de aplicações na área climatológica é ainda pequeno e podese citar os trabalhos recentes dos autores Schwartz et al. (2013), Singh e Sarkar (2013) e Mazucheli e Emanuelli (2015), sendo que apenas neste último a distribuição foi ajustada a dados referentes ao volume de precipitação.

Em geral, várias distribuições concorrentes podem ser propostas para a explicação de um mesmo fenômeno. A escolha da distribuição mais adequada devese pautar na comparação de medidas que avaliam a qualidade do ajuste, que são denominadas de goodness-of-fit (GOF), como o valor da estatística do teste de Kolmogorov-Smirnov (KS). Estas medidas quantificam o quão bem a distribuição ajusta-se a um conjunto de observações, e podem ser usadas para comparar o ajuste de distribuições concorrentes (SCHULTHEIS; NAIDU, 2014). No entanto, existe uma tendência para a seleção de distribuições complexas, mesmo que distribuições mais simples sejam mais parcimoniosas (PITT; MYUNG, 2002).

Para contornar este problema surgiu o conceito de mimetismo, o qual quantifica o quão bem os modelos são capazes de imitar uns aos outros, ou seja, a ENCICLOPÉDIA BIOSFERA, Centro Científico Conhecer - Goiânia, v.14 n.26; p.1217 
habilidade de cada distribuição fornecer bons ajustes aos dados obtidos pela outra distribuição (SCHULTHEIS; NAIDU, 2014). Para quantificar o mimetismo, existe o procedimento denominado de PBC (Parametric Bootstrap Cross-Fitting) que gera duas distribuições a partir das subtrações de uma especificada medida de GOF, esperadas sob cada uma das distribuições concorrentes, sendo tais distribuições obtidas por meio do método Bootstrap, introduzido por Efron (1979). Entretanto, ao realizar uma busca na literatura, não foram encontradas aplicações do método PBC para a discriminação entre distribuições usadas no estudo de variáveis climatológicas.

No trabalho em que propõe o método PBC, Wagenmakers et al. (2004) apresentam duas versões do mesmo, que diferem-se quanto a forma de gerar os dados, sendo a primeira indicada para avaliar o ajuste e mimetismo da distribuição para um banco de dados especificado e a segunda indicada para extrair conclusões mais genéricas sobre o ajuste das distribuições em questão.

Conforme Wagenmakers et al. (2004), dado o valor de alguma estatística de GOF (como a $K S$ ), a versão do PBC que depende de um determinado conjunto de dados é aplicado adotando os passos a seguir: (1) Gerar uma amostra Bootstrap não paramétrica, denotada por $x^{*}$, dos dados originais $x$; (2) Ajustar as distribuições $A$ e $B$ a amostra $x^{*}$, obtendo os EMV dos vetores $\hat{\theta}_{A}^{*}$ e $\hat{\theta}_{B}^{*}$ de parâmetros, respectivamente; (3) Simular dados segundo a distribuição $A\left(D\left(\hat{\theta}_{A}^{*}\right)\right)$ e a distribuição $B\left(D\left(\hat{\theta}_{B}^{*}\right)\right)$, por meio do Bootstrap paramétrico; (4) Ajustar as distribuições $A$ e $B$ a $D\left(\hat{\theta}_{A}^{*}\right)$, e obter a diferença $\left(\triangle G O F_{A B}^{*} \mid A=G O F_{A}^{*}-G O F_{B}^{*}\right)$ de GOF entre os dados simulados pela distribuição $A$. (5) Ajustar as distribuições $A$ e $B$ a $D\left(\hat{\theta}_{B}^{*}\right)$ e obter a diferença $\left(\triangle G O F_{A B}^{*} \mid B=G O F_{A}^{*}-G O F_{B}^{*}\right)$ de GOF entre os dados simulados da distribuição $B$; (5) Realizar as etapas anteriores $M$ vezes.

Desta forma, a aplicação do PBC resulta em um vetor de diferenças de GOF sob a distribuição $A$ e uma sob a distribuição $B$. Schultheis e Singhaniya (2013) apontam que a utilidade do PBC depende essencialmente de uma utilização adequada das distribuições de diferença de GOF (denotadas por $\triangle G O F_{A B}^{*}$ ) geradas para selecionar entre os modelos concorrentes.

A quantidade $\triangle G O F_{A B}^{*} \mid A$ permite medir o quão provável é obter a diferença GOF observada $\left(\delta_{A B}\right)$ ajustando as distribuições aos dados observados se a variável de interesse segue a distribuição $A$. A quantidade $\triangle G O F_{A B}^{*} \mid B$ permite medir o quão provável é obter $\delta_{A B}$ se a distribuição $B$ é o modelo de geração (SCHULTHEIS; NAIDU, 2014). Assim, a probabilidade de que a variável de interesse segue a distribuição $A$, em vez da distribuição $B$, dada a diferença observada de GOF, pode ser quantificada pela razão entre as estimativas da altura das distribuições $\triangle G O F_{A B}^{*} \mid A$ e $\triangle G O F_{A B}^{*} \mid B$ no valor $\delta_{A B}$ :

$$
P\left(\delta_{A B} \mid A\right) / P\left(\delta_{A B} \mid B\right)=P_{A}(x) / P_{B}(x) .
$$

De acordo com Wagenmakers et al. (2004), o critério para decisão da escolha entre os modelos denominado ótimo, definido como o critério que maximiza a probabilidade de uma classificação binária correta, é calculado por $P_{A}(x) / P_{B}(x)=1$. $O$ viés causado pelo mimetismo $\left(\beta_{m}\right)$ é quantificado pela subtração entre o critério nominal, $\triangle G O F_{A B}=0$ e o ótimo (WAGENMAKERS et al., 2004).

Considerando a importância de explicar o comportamento dos volumes de precipitação mensal; da possibilidade do uso do método PBC para comparação do ENCICLOPÉDIA BIOSFERA, Centro Científico Conhecer - Goiânia, v.14 n.26; p.1218 2017 
ajuste de duas distribuições concorrentes para explicar a complexidade das distribuições, e do método PBC não ter sido utilizado em modelos de variáveis climatológicas de acordo com a revisão da literatura, objetivou-se neste trabalho realizar a discriminação e a comparação do mimetismo das distribuições Gama e a Nakagami, avaliandas por meio da aplicação da variação do PBC ajustadas aos dados referentes as precipitações mensais observadas na estação meteorológica convencional de Maringá - PR.

\section{Os dados}

\section{MATERIAL E MÉTODOS}

Foram utilizados dados da estação meteorológica convencional da cidade Maringá - PR (Figura 1), compilados a partir das séries históricas de precipitação mensais obtidas no Banco de Dados Meteorológicos para Ensino e Pesquisa (BDMEP) do Instituto Nacional de Meteorologia, o INMET (<www.inmet.gov.br/portal/index.php? $r=b d m e p / b d m e p>$ ), órgão responsável pela coleta e disponibilização das informações meteorológicas oficiais.

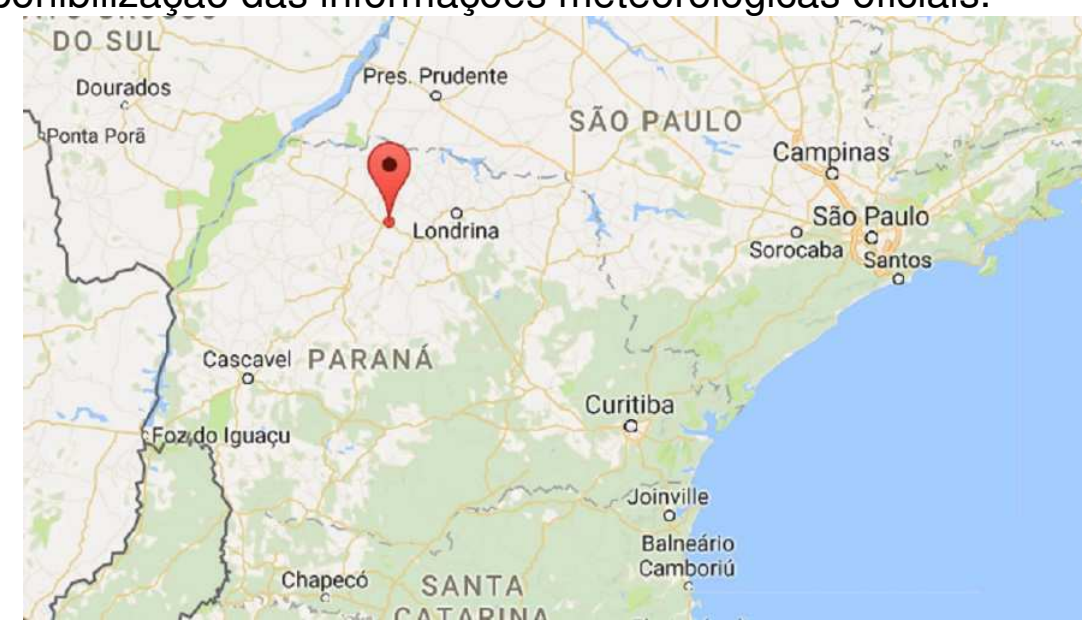

FIGURA 1. Localização da estação meteorológica convencional de Maringá - PR. Fonte: Google Maps (2017).

Considerou-se como variável de estudo o total de precipitação acumulada no mês, a fim de realizar a comparação entre duas distribuições propostas (Gama e Nakagami) para a explicação de tal fenômeno. Nos dados obtido do INMET encontram-se disponíveis, de forma digital, apenas os registros mensais a partir do ano de 1961. Entretanto, foram selecionadas as séries históricas disponíveis no período entre janeiro de 1964 e dezembro de 2016, já que entre os anos de 1961 e 1963 os registros da estação convencional de Maringá foram realizados apenas para alguns meses.

As informações referentes aos volumes de precipitação mensais não estavam disponíveis para todos os meses do período considerado, sendo que no total, foram utilizadas 527 observações, divididas em 12 séries mensais para o ajuste das distribuições.

\section{As distribuições}

Para análise da pluviosidade total mensal, utilizou-se a distribuição Gama, comum no estudo de variáveis de precipitação, e a Nakagami. A seguir descreve-se as distribuições objeto de estudo do presente artigo.

\section{- Distribuição de Nakagami:}

Uma variável aleatória não negativa $X$ com distribuição Nakagami tem, respectivamente, função de densidade e de distribuição escritas nas formas: 


$$
f(x \mid \Theta)=\frac{2}{\Gamma(\alpha)}\left(\frac{\alpha}{\theta}\right)^{\alpha} x^{2 \alpha-1} \exp \left[-\frac{\alpha}{\theta} x^{2}\right]
$$

e

$$
F(x \mid \Theta)=\Gamma\left(\frac{\alpha}{\theta} x^{2}, \alpha, 1\right)
$$

em que $\Theta=(\theta, \alpha)$ é o vetor de parâmetros, $\theta>0$ o parâmetro de escala, $\alpha \geq 0.5$ o parâmetro de forma, $\Gamma(\cdot)$ a função gama e $\Gamma(\cdot, a, b)$ é a função de distribuição acumulada de uma variável aleatória com distribuição Gama com parâmetro de forma $a$ e escala $b$. Para $\alpha=1$ tem-se a distribuição Half-Normal e para $\alpha=0.5$ a distribuição Rayleigh. Por meio do método de transformação de variáveis aleatórias mostra-se facilmente que se $X \sim \operatorname{Nakagami}(\theta, \alpha)$ então $Y=X^{2}$ tem distribuição Gama com parâmetro escala $\frac{\theta}{\alpha}$ e forma $\alpha$.

\section{- Distribuição Gama:}

Uma variável aleatória não negativa $X$ com distribuição Gama tem, respectivamente, função de densidade e de distribuição escritas nas formas:

$$
f(x \mid \Theta)=\frac{1}{\theta^{\alpha \Gamma(\alpha)}} x^{\alpha-1} \exp \left(-\frac{x}{\theta}\right)
$$

e

$$
F(x \mid \Theta)=\int_{0}^{x} \frac{1}{\theta^{\alpha \Gamma(\alpha)}} x^{\alpha-1} \exp \left(-\frac{x}{\theta}\right)
$$

em que $\Theta=(\theta, \alpha)$ é o vetor de parâmetros, $\theta>0$ o parâmetro de escala, $\alpha>0$ o parâmetro de forma e $\Gamma(*)$ a função gama. Para $\alpha=1$, tem-se a distribuição exponencial e, para $\alpha=n / 2$ e $\theta=2$, a distribuição qui-quadrado com $n$ graus de liberdade.

\section{Aplicação do método PBC}

Para realizar a aplicação do método PBC, utilizou-se as séries históricas de precipitação mensais da estação convencional de Maringá. Foram realizadas $\mathrm{M}=$ 1000 iterações, sendo assim, as densidades empíricas das distribuições de $\triangle G O F_{A B}^{*}$ foram estimadas a partir de 1000 valores. Utilizou-se a estatística KS como medida de GOF, dada por:

$$
K S=\max _{1 \leq i \leq n}\left(\widehat{z_{l}}-\frac{i}{n}, \frac{i}{n}-\widehat{z_{l}}\right), \text { para } i=1, \ldots, n
$$

Para descrever dados de precipitações, foram selecionadas as distribuições Gama e Nakagami, comparadas por meio do DIPBC. É importante enfatizar que a Gama é a distribuição usada pelo INMET para a modelagem da precipitação. $O$ algoritmo para aplicação do método de discriminação foi implementado no ambiente estatístico R (R CORE TEAM, 2016), assim como todas as análises realizadas. 


\section{RESULTADOS E DISCUSSÃO}

Os resultados apresentados na Tabela 1 mostram que os maiores volumes são observados entre os meses de dezembro e fevereiro, chegando a uma precipitação média de $204 \mathrm{~mm}$ no mês de janeiro. Em contrapartida, os meses de inverno (junho a agosto) foram aqueles em que os menores volumes de precipitação média foram observados, e para tais meses a precipitação mínima foi de $0 \mathrm{~mm}$, indicando que houveram anos em que não ocorreu precipitação nos meses em questão.

TABELA 1. Medidas resumo das séries históricas de precipitação pluviométrica agrupadas por mês, da estação meteorológica convencional de Maringá - PR, de 1964 a 2016.

\begin{tabular}{lccccccc}
\hline \multicolumn{1}{c}{ Mês } & n & Média & DP & CV & Mediana & Mínimo & Máximo \\
\hline Janeiro & 45 & 204,01 & 91,83 & $45 \%$ & 205,10 & 27,60 & 419,80 \\
Fevereiro & 45 & 191,30 & 99,66 & $52 \%$ & 168,50 & 46,20 & 426,00 \\
Março & 45 & 152,12 & 74,05 & $49 \%$ & 130,50 & 33,60 & 340,00 \\
Abril & 44 & 115,35 & 63,82 & $55 \%$ & 104,75 & 1,00 & 346,30 \\
Maio & 43 & 115,99 & 93,56 & $81 \%$ & 89,70 & 0,70 & 396,40 \\
Junho & 43 & 105,21 & 93,09 & $88 \%$ & 92,80 & 0,00 & 396,70 \\
Julho & 43 & 70,53 & 69,83 & $99 \%$ & 52,10 & 0,00 & 378,60 \\
Agosto & 42 & 53,91 & 52,14 & $97 \%$ & 38,95 & 0,00 & 219,80 \\
Setembro & 45 & 119,01 & 79,41 & $67 \%$ & 94,00 & 21,40 & 319,60 \\
Outubro & 43 & 164,32 & 82,83 & $50 \%$ & 142,10 & 45,00 & 345,60 \\
Novembro & 44 & 138,17 & 83,31 & $60 \%$ & 114,45 & 26,20 & 369,60 \\
Dezembro & 45 & 176,64 & 72,28 & $41 \%$ & 176,80 & 45,00 & 360,40 \\
\hline
\end{tabular}

* DP: Desvio padrão; CV: Coeficiente de variação. Fonte: Autores.

Observa-se uma grande variabilidade na distribuição dos volumes de precipitação, com coeficientes de variação chegando a $99 \%$ para o mês de julho. Observa-se que em geral, as distribuições dos volumes mensais de chuva (Figura 2) apresentam assimetria à direita, com medianas inferiores as respectivas médias de precipitação. Também nota-se a presença de alguns valores atípicos, sobretudo para os meses entre abril e julho.

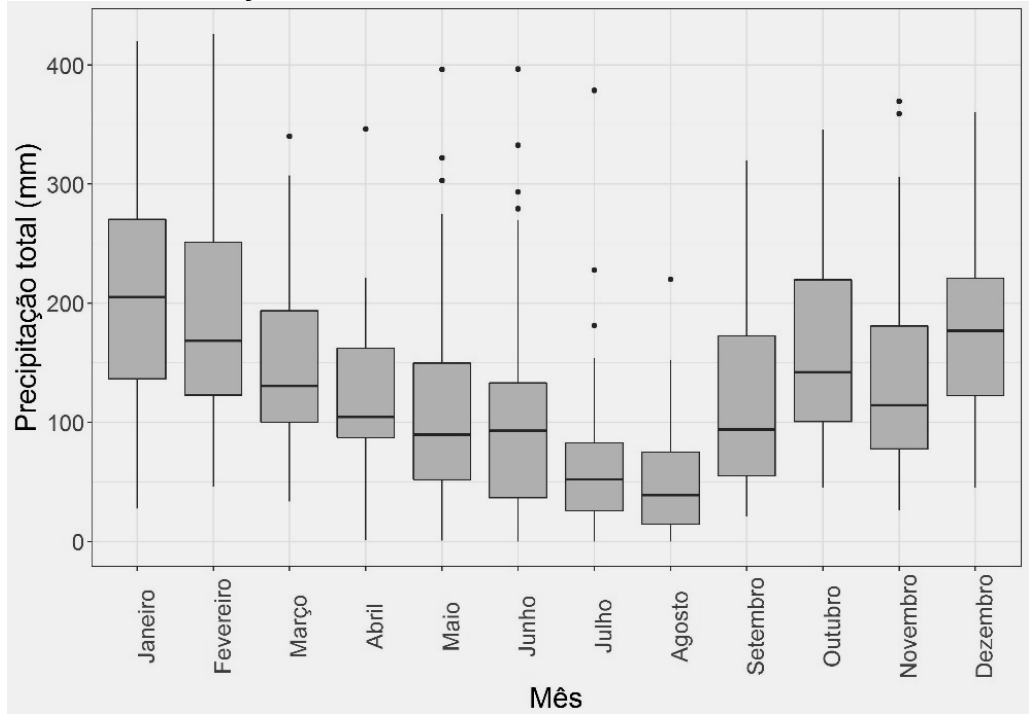

FIGURA 2. Box-plot das séries históricas de precipitação pluviométrica agrupadas por mês, da estação meteorológica convencional de Maringá - PR, de 1964 a 2016. Fonte: Autores (2017) 
O algoritmo do método DIPBC, foi aplicado as séries históricas de volumes de precipitação de cada mês da estação convencional de Maringá no período considerado, resultando em 12 conjuntos de dados. Observa-se na Figura 3 que na maior parte dos meses observados, a distribuição Gama foi mais flexível para o ajuste aos dados obtidos pelo modelo concorrente, apresentando maior mimetismo. Também observa-se que para três quartos dos meses observados, o PBC indicou que a distribuição Nakagami se ajusta melhor aos dados, e além disso, nos meses de Junho e Julho, a análise isolada do KS apontou uma conclusão contrária.

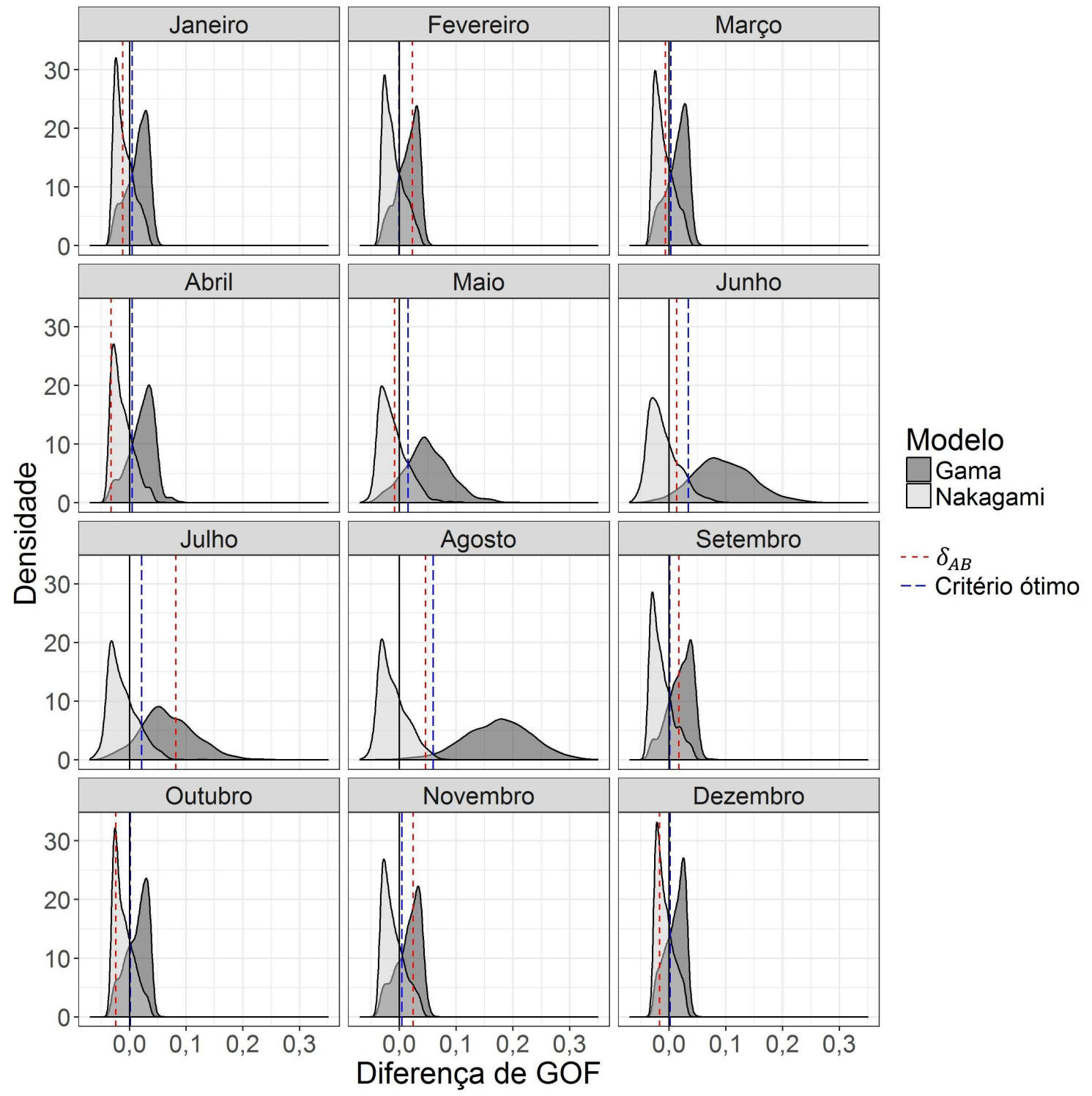

FIGURA 3. Distribuições das diferenças de GOF obtidas pelo DIPBC aos dados mensais de precipitação pluviométrica da estação meteorológica convencional de Maringá - PR, de 1964 a 2016, para comparação das distribuições Gama e Nakagami. Fonte: Autores.

Ao contrário da distribuição Gama, uma das mais utilizadas na modelagem de dados climáticos, ainda é pequeno o número de aplicações da Nakagami a este tipo de variáveis, sendo que em geral, a mesma é utilizada na área de engenharia de comunicações (KARAGIANNIDIS et al., 2007). Ressalta-se que qualquer distribuição de probabilidade, com suporte nos números reais positivos, pode ser utilizada na 
descrição do comportamento de séries climatológicas, mas em se tratando de séries de pluviosidade total diária,decendial, mensal, entre outras, as distribuições Weibull e Gama são as mais utilizadas (MAZUCHELI; EMANUELLI, 2015).

No presente estudo, observou-se que em oito das 12 séries históricas consideradas, o critério de decisão do método PBC apontou que a Nakagami é a distribuição mais plausível para a descrição do comportamento da variável de interesse. Ramos e Moala (2014) também compararam distribuições utilizadas frequentemente em climatologia (Gama, Weibull e Lognormal), com uma distribuição não usual (Exponencial Geométrica Estendida), mostrando que distribuições menos usuais podem ser aplicadas com sucesso em dados referentes a precipitação.

Um estudo recente, na análise da pluviosidade total mensal, determinou que a Nakagami foi a distribuição mais apropriada em $34,43 \%$ das séries seguida por $29,72 \%$ e $22,88 \%$ pelas distribuições Weibul e Gama (MAZUCHELI; EMANUELLI, 2015). Outra modelagem de dados de precipitação pluviométrica mensal apresentou resultados indicando que a distribuição Gama ajustou-se mais adequadamente que as distribuições log-normal e Weibull (RODRIGUES et al., 2013). Em dados de chuva total anual, além da Gama, foram encontrados na literatura ajustes satisfatórios para as distribuições Gumbel, Normal e Weibull (SILVA et al., 2013). No entanto, nenhum deste trabalhos testou a característica de mimetismo visando mostrar os modelos mais simplistas. No presente estudo testou-se exatamente isso.

Com os dados observados foi possível notar, que o viés causado pelo mimetismo $\beta_{m}$ foi relativamente baixo para a maior parte dos meses, sendo que os maiores valores de $\beta_{m}$ foram observados para os dados referentes a junho e agosto, meses nos quais o PBC indicou a distribuição, contrário ao apontado pela estatística KS isoladamente, favorecendo a escolha pela Nakagami. Como comentado anteriormente, a estatística $K S$, uma medida de GOF, não considera a complexidade funcional das distribuições para selecioná-las, tendendo a escolher distribuições mais complexas.

De um modo geral, distribuições complexas serão capazes de imitar distribuições mais simples. No entanto, o aumento da complexidade não aumenta necessariamente 0 mimetismo. Assim, o DIPBC só pode discriminar entre as distribuições na medida em que estes são funcionalmente diferentes. Se uma distribuição é estruturalmente mais complexa, mas funcionalmente idêntica a uma distribuição mais simples, a seleção por esse método será potencialmente enganosa (WAGENMAKERS et al., 2004).

As áreas sobrepostas entre as distribuições de $\triangle G O F_{A B}^{*}$, que são delimitadas pelo critério definido como ótimo, representam a habilidade de mimetismo de cada distribuição. Assim, observa-se que pelos resultados do PBC, a distribuição Gama exibiu maior flexibilidade na explicação dos dados provenientes da Nakagami do que o contrário. Ressalta-se que os resultados gerados pelo método são específicos aos conjuntos de observações analisadas, já que a versão DIPBC foi utilizada.

O DIPBC pode ser interpretado como uma implementação frequentista do método BPP (Bayesian posterior predictive $p$-values), usualmente utilizado para avaliar o ajuste de distribuições. Ambos os métodos geram distribuições de valores de GOF esperados para as distribuições sob consideração, que podem ser usadas para avaliar o mimetismo ou a adequação da distribuição. Entretanto, no procedimento BPP, a distribuição para gerar os dados simulados é explicitamente Bayesiano (WAGENMAKERS et al., 2004). 
Se a distribuição dos parâmetros respectivos aos modelos obtida pelo Bootstrap não paramétrico é idêntica a distribuição à posteriori Bayesiana dos parâmetros, os dois métodos produzem os mesmos resultados, sendo que a distribuição não paramétrica tem vantagens práticas. Perturbando os dados, o Bootstrap aproxima do efeito Bayesiano de perturbação dos parâmetros, sendo normalmente muito mais simples de se realizar (HASTIE et al., 2001).

\section{CONCLUSÃO}

O desenvolvimento do presente trabalho permitiu a avaliação da utilização de um método recente de discriminação de distribuições, o PBC, para o ajuste de variáveis climatológicas, área na qual o método ainda não foi explorado, de acordo com pesquisa na literatura. Embora a distribuição Nakagami não seja uma distribuição usualmente utilizada para o ajuste de variáveis climatológicas, por meio da aplicação do método PBC a dados de precipitação reais, foi verificado que a mesma apresenta melhor ajuste se comparada a distribuição Gama para a maior parte dos meses considerados e que em geral, a distribuição Gama apresenta maior complexidade funcional em relação a Nakagami, com maior viés de mimetismo para todas as séries históricas consideradas.

Ainda, os resultados da aplicação do método indicaram a importância da consideração do mimetismo na discriminação entre distribuições, uma vez que justamente para os casos em que foram observados os maiores valores de viés de mimetismo, o PBC apontou uma conclusão contrária a sugerida pela medida de qualidade de ajuste utilizada, a estatística KS. Tais constatações recomendam o método PBC para confrontar duas distribuições levando em consideração as respectivas complexidades funcionais, se comparado aos critérios de GOF usuais. Entretanto, é preferível que a discriminação entre distribuições seja baseada na aplicação de ambas as técnicas, já que estas avaliam diferentes questões.

Diante da utilidade do PBC apresentada neste trabalho, torna-se evidente a necessidade de sua exploração e aplicação em diversos contextos, sobretudo em climatologia.

\section{AGRADECIMENTOS}

Agradecemos ao Instituto Nacional de Meteorologia (InMet) por deixar disponíveis no portal (http://www.inmet.gov.br) os dados analisados neste trabalho. Agradecemos também ao $\mathrm{CNPq} / \mathrm{FA} / \mathrm{UEM}$, pelo apoio financeiro ao primeiro autor. $\mathrm{O}$ terceiro autor agradece ao Instituto Cesumar de Ciência, Tecnologia e Inovação (ICETI) pela Bolsa de Produtividade em Pesquisa concedida.

\section{REFERÊNCIAS}

BEYRUTH, Z. Água, agricultura e as alterações climáticas globais. Revista Tecnologia \& Inovação Agropecuária, n. 6, p. 74-89. 2008. Disponível em: $<$ www.apta.sp.gov.br/Publicacoes/T\&IA/T\&IAv1n1/Revista_Apta_Artigo_Agua_Agric ultura.pdf>.

CHOW, V.; MAIDMENT, D.; MAYS, L. Applied Hydrology, 2nd Edition. McGrawHill, 2013.

COE, R.; STERN, R. D. Fitting models to daily rainfall data. Journal of Applied Meteorology, v. 21, n. 7, p. 1024-1031, 1982. Disponível em: 
<doi.org/10.1175/1520-0450(1982)021<1024:FMTDRD>2.0.CO;2>.

doi:

10.1175/1520-0450.

DOURADO NETO D.; ASSIS, J. P.; TIMM, L. C.; MANFRON, P. A.; SPAROVEK, G.; et. al. Goftest: Classical Goodness-of-Fit Tests for Univariate Distributions. $R$ package version 1.0-2. 2014. Disponível em: <http://CRAN.Rproject. org/package $=$ goftest $>$.

EFRON, B. Bootstrap methods: another look at the jackknife. The annals of Statistics, p. 1-26, 1979. Disponível em: <dx.doi.org/10.1214/aos/1176344552>. doi: $10.1214 /$ aos/1176344552.

HASTIE, T.; TIBSHIRANI, R.; FRIEDMAN, J. The Elements of Statistical Learning: Data Mining, Inference, and Prediction. Springer New York, 2001.

KARAGIANNIDIS, G. K.; SAGIAS, N. C.; MATHIOPOULOS, P. T. NxNakagami: A Novel Stochastic Model for Cascaded Fading Channels. IEEE Transactions on Communications, v. 55, n. 8, p. 1453-1458, 2007. Disponível em: <doi.org/10.1109/TCOMM.2007.902497>. doi: 10.1109/TCOMM.2007.902497.

MAZUCHELI, J.; EMANUELLI, I. P. Aplicação da distribuição nakagami na análise de dados de precipitação. In: $60^{\underline{a}}$ Reunião Anual da Região Brasileira da Sociedade Internacional de Biometria (RBras) e 16은 Simpósio de Estatística Aplicada a Experimentação Agronômica (SEAGRO), Presidente Prudente, 2015.

MEYLAN, P.; FAVRE, A.; MUSY, A. Predictive Hydrology: A Frequency Analysis Approach. CRC Press, 2012.

NAKAGAMI, M. The m-distribution-A general formula of intensity distribution of rapid fading. Statistical Method of Radio Propagation, p. 3-34, 1960. Disponível em: <doi.org/10.1016/B978-0-08-009306-2.50005-4>. doi: 10.1016/B978-0-08-0093062.50005-4.

PITT, M. A.; MYUNG, J. When a good fit can be bad. Trends in cognitive sciences, v. 6, n. 10, p. 421-425, 2002. Disponível em: <dx.doi.org/10.1016/S13646613(02)01964-2>. doi: 10.1016/S1364-6613(02)01964-2.

RAMOS, P. L.; MOALA, F. A. The extended geometric exponential distribution applied for modeling rainfall data. Revista Brasileira de Meteorologia, v. 29, n. 4, 2014. Disponível em: <dx.doi.org/10.1590/0102-778620130612>. doi: 10.1590/0102778620130612.

R CORE TEAM. R: A Language and Environment for Statistical Computing. R Foundation for Statistical Computing, Vienna, Austria, 2016. Disponível em: <https://www.R-project.org/>. Acesso em: 30 de mai. 2016.

RODRIGUES, J. A.; DOS SANTOS FILHO, J.; CHAVES, L. M. Funções densidade de probabilidade para a estimativa de precipitação mensal. Semina: Ciências Exatas e Tecnológicas, v. 34, n. 1, p. 3-8, 2013. Disponível em: <dx.doi.org/10.5433/1679-0375.2013v34n1p3>. doi: 10.5433/1679-0375. 
SCHULTHEIS, H.; NAIDU, P. Multi-model comparison using the cross-fitting method. In: Proceedings of the 36th annual meeting of the Cognitive Science Society, Austin, p. 1389 - 1394, 2014.

SCHULTHEIS, H.; SINGHANIYA, A. Decision criteria for model comparison using cross-fitting. Cognitive Systems Research, v. 33, p. 100-121, 2013. Disponível em: $<$ dx.doi.org/10.1016/j.cogsys.2014.09.003>. doi: 10.1016/j.cogsys.2014.09.003.

SCHWARTZ, J.; GODWIN, R. T.; GILES, D. E. Improved maximum-likelihood estimation of the shape parameter in the Nakagami distribution. Journal of Statistical Computation and Simulation, v. 83, n. 3, p. 434-445, 2013. Disponível em: $\quad$ <dx.doi.org/10.1080/00949655.2011.615316>.

doi: 10.1080/00949655.2011.615316.

SILVA, I. N.; DE OLIVEIRA, J. B.; FONTES, L. O.; ARRAES, F. D. D. Distribuição de frequência da chuva para região Centro-Sul do Ceará, Brasil. Revista Ciência Agronômica, v. $44, \quad$ n. 3 , p. 481, 2013. Disponível em: <ccarevista.ufc.br/seer/index.php/ccarevista/article/view/2140/825>.

SINGH, K. J.; SARKAR, S. Development of GIUH for the catchment contributing to Loktak lake, North East India. Journal of the Indian Society of Remote Sensing, v. 41, n. 2, p. 447-459, 2013. Disponível em: <dx.doi.org/ 10.4236/jep.2011.26090>. doi: 10.4236/jep.2011.26090.

WAGENMAKERS, E.; RATCLIFF, R.; GOMEZ, P.; IVERSION,G. J. Assessing model mimicry using the parametric Bootstrap. Journal of Mathematical Psychology, $v$. 48, n. 1, p. 28-50, 2004. Disponível em: <dx.doi.org/ 10.1016/j.jmp.2003.11.004>. doi: 10.1016/j.jmp.2003.11.00. 